\title{
The association of Metabolic Syndrome and its Components with the Incidence and Survival of Colorectal Cancer: A Systematic Review and Meta-analysis
}

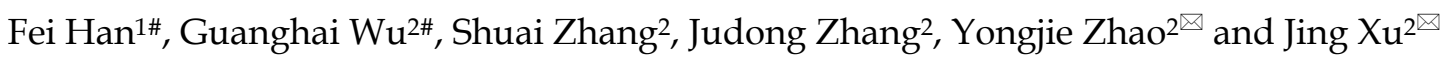

1. NHC Key Laboratory of Hormones and Development, Tianjin Key Laboratory of Metabolic Diseases, Chu Hsien-I Memorial Hospital \& Tianjin Institute of Endocrinology, Tianjin Medical University, Tianjin 300134, China.

2. Department of General Surgery, Tianjin Union Medical Center, Tianjin 300121, China.

\#These authors contributed equally to this work.

$\triangle$ Corresponding authors: Equal contributions of co-corresponding authors. Yongjie Zhao MD, PhD, E-mail: zhaoyongjie@sohu.com; Tel.: +86-22-27557221. Jing Xu, MD, PhD, E-mail: xujingdoc@126.com; Tel.: +86-22-27557221; Department of General Surgery, Tianjin Union Medical Center, Jieyuan Road 190, Hongqiao District, Tianjin, 300121, PR China; Fax: +86-22-27557221.

(C) The author(s). This is an open access article distributed under the terms of the Creative Commons Attribution License (https://creativecommons.org/licenses/by/4.0/). See http://ivyspring.com/terms for full terms and conditions.

Received: 2020.08.26; Accepted: 2020.11.13; Published: 2021.01.01

\begin{abstract}
Background: This meta-analysis was aimed to quantitatively assess the associations of metabolic syndrome (MetS) and its components with colorectal cancer (CRC).

Methods: PubMed, EMBASE and Web of Science databases were systematically searched for eligible studies. A total of 18 studies for CRC incidence and 12 studies for CRC mortality were identified.

Results: MetS was associated with an increased risk of CRC incidence and mortality in male (RR: 1.28, 95 $\% \mathrm{Cl}$ 1.16-1.39, and 1.24, 1.18-1.31, respectively) and correlated with an increased risk of CRC incidence in female (RR: 1.21, 1.13-1.30), but not with CRC mortality in female. MetS increased the risk of cancer-specific mortality (RR: 1.72, 1.03-2.42), but not overall mortality. The risk estimates of CRC incidence changed little depending on age, sex, cancer site, the type of studies, ethnicity, publication year, or definition of MetS. As for CRC mortality, further stratified analyses indicated statistical significance in studies with assessing cancer-specific survival outcome, in male, a cohort design, ethnicity of non-Chinese or with definition of MetS as $\geq 3$ metabolic abnormalities. Obesity and hyperglycemia are risk factors of CRC incidence in both male and female. Only dysglycemia is the risk factor for CRC mortality.

Conclusions: MetS is associated with an increased risk of CRC incidence and cancer-specific mortality, but not overall mortality. In addition, MetS may increase the CRC mortality in male rather than in female. However, since most of the studies on CRC mortality were conducted in Chinese, further studies are needed to clarify this connection.
\end{abstract}

Key words: metabolic syndrome; colorectal cancer; incidence; survival; meta-analysis

\section{Introduction}

Metabolic syndrome (MetS) is a cluster of metabolic risk factors that includes abdominal obesity, hypertension, hyperglycemia, and dyslipidemia [1]. The prevalence of MetS ranges between $34.8 \%$ and $41.9 \%$ in the US and $18 \%$ and $46 \%$ in Europe [2,3]. The number varies depending on race, environmental factors, genetic differences, physical activity level, eating habits, and differences in measurement standards [4]. However, the rapid growing of MetS prevalence raises lots of public health concerns including cancer.

Nowadays, given the rising prevalence of MetS all over the world and the high occurrence of cancers, especially colorectal and breast cancers, many cases of 
cancer may be linked to MetS $[5,6]$. MetS and cancer share many modifiable risk factors including age, genetic factors, obesity, physical inactivity, unhealthy diet, alcohol and smoking [7]. MetS has been closely linked to cancer, as it increases cancer risk and cancerrelated mortality. Moreover, MetS usually occurs as a consequence of specific chemotherapy drugs and radiotherapy; therefore, MetS and diabetes mellitus have been increasingly recognized as long-term complications of childhood cancer treatment [8]. Hence, cancer survivors have an increased risk of MetS [7]. So, clarifying the underlying mechanisms linked MetS to cancer is important to prevent or delay these two conditions.

Colorectal cancer (CRC) is the third most commonly diagnosed cancer worldwide and one of the leading causes of cancer-specific death [9], with more than 1.1 million cancer deaths expected by 2030 [10]. According to the published studies, diet [11], obesity [12], alcohol intake [13] and diabetes [14] are all risk factors for the occurrence and mortality in patients with CRC. Since 2001, several epidemiological studies have investigated the association between MetS and CRC risk but showed inconsistent results. As some studies showed that, MetS plays an important role in CRC [15-17]. However, there were also studies showed no significant correlation between the two [18-22]. In the above studies, MetS may increase the risk of CRC in male rather than female $[18,20]$. In addition, the correlation may also differ because of cancer site [23]. Apart from increasing the cancer risk, studies also reported that MetS is an important risk factor for cancer mortality. It is reported that patients with MetS has a higher mortality [24, 25]. However, some studies declared that MetS has no effect on CRC mortality [26, 27]. Many studies also discussed the role of different MetS components in the development and progression of CRC. Most of them showed that obesity and diabetes may be risk factors for CRC $[19,25]$, while some are not [21, 27].

In view of the ambivalent results listed above, clarifying the association of MetS and its components with the incidence and survival of colorectal cancer, may help revealing important risk and prognostic factors of CRC. Based on the results, clinicians can also make effective strategies to prevent the onset and development of CRC. Esposito et al. carried out a meta-analysis to unearth the correlation between MetS and its components with the progression of CRC seven years ago [28], however, a lot of new clinical research were conducted after that. Due to the small numbers of studies and patients included, this previous review might not fully explore the potential variation of this association. As data on the relation between MetS and CRC occurrence and survival are accumulating lately, we therefore decided to synthesize the results of published studies to test whether MetS and its individual components can predict risk and outcomes in patients with CRC.

\section{Methods}

\section{Search strategy}

PubMed, EMBASE databases and Web of Science were searched from inception to May 31st, 2020, for eligible studies on the relationship between MetS and CRC. The terms used to retrieve literatures were the following: Colorectal OR colon OR rectal AND cancer OR carcinoma OR malignancy OR tumor OR neoplasm AND metabolic syndrome AND risk OR incidence OR survival $O R$ prognosis $O R$ mortality. We also referred to the reference lists from reviews or relevant papers to get more eligible researches. Conference abstracts were also included if sufficient data were provided. There was no language restriction. Two authors independently performed the literature search and identified potential studies of the title, abstract and full-text.

\section{Selection criteria}

Reports were included if they met the criteria as follows: (1) study designs: case-control studies, cohort studies and randomized controlled trials (RCTs); (2) risk estimates of CRC incidence or mortality with $95 \%$ CIs (Confidences Intervals) were reported. If the same data were used in several studies, we selected the publication with the largest number of cases or more details. The exclusion criteria: (1) letters, editorials, abstracts, reviews, case reports or expert opinions; (2) studies not based on people; (3) outdated articles with little significance or credibility. Literature search also was independently done by two authors (F.H. and G.W.).

\section{Data Extraction}

From each included study, data were independently extracted by two investigators (F.H. and G.W.) using a standardized data extraction form. Briefly, we recorded study characteristics including first author name, publication year, country, average/range of age, mean/median duration of follow-up, specific outcomes, total number of individuals, number of cases, and risk estimates and their $95 \%$ CIs. In order to dissect the influence of any single component of the metabolic syndrome, risk estimates for each single component were collected. Disagreements between investigators were discussed and resolved by an additional reviewer. 


\section{Quality Assessment}

Study quality was independently assessed by two of us (F.H. and G.W.). Quality of the included studies was evaluated by use of the Newcastle Ottawa Scale (NOS). According to its criteria, studies were assessed on the basis of three perspectives: selection, comparability and outcomes. The full score was defined as 9 stars, and a study was classified as low, moderate and high-quality using 0-3, 4-6 and 7-9 stars, respectively. Differences were resolved by discussion. PRISMA (preferred reporting items for systematic reviews and meta-analyses) checklist was followed for reporting systematic reviews and meta-analyses [29].

\section{Statistical analysis}

In a conservative approach, the random-effects estimates of relative risk (RR), which allow for variation of true effects across studies, were taken as "main results" [28]. Statistical heterogeneity among studies was evaluated with the use of $I^{2}$ statistic. Significant heterogeneity was assumed for $I^{2}>50 \%$ or a $Q$ test $p$-value $<0.05$ [30]. We utilized the random-effects model to combine RRs from single studies if obvious heterogeneity was observed [31]. Subgroup analyses were conducted to explore potential sources of heterogeneity across studies. In the sensitivity analysis, studies were omitted one by one and the others were analyzed to evaluate the effect of a single study on the summary risk estimates. Publication bias was assessed statistically with Kendall's tau $[32,33]$. A $p$-value $<0.05$ in these tests suggests the presence of publication bias. We utilized STATA (Version 12.0, College Station, TX, USA) to

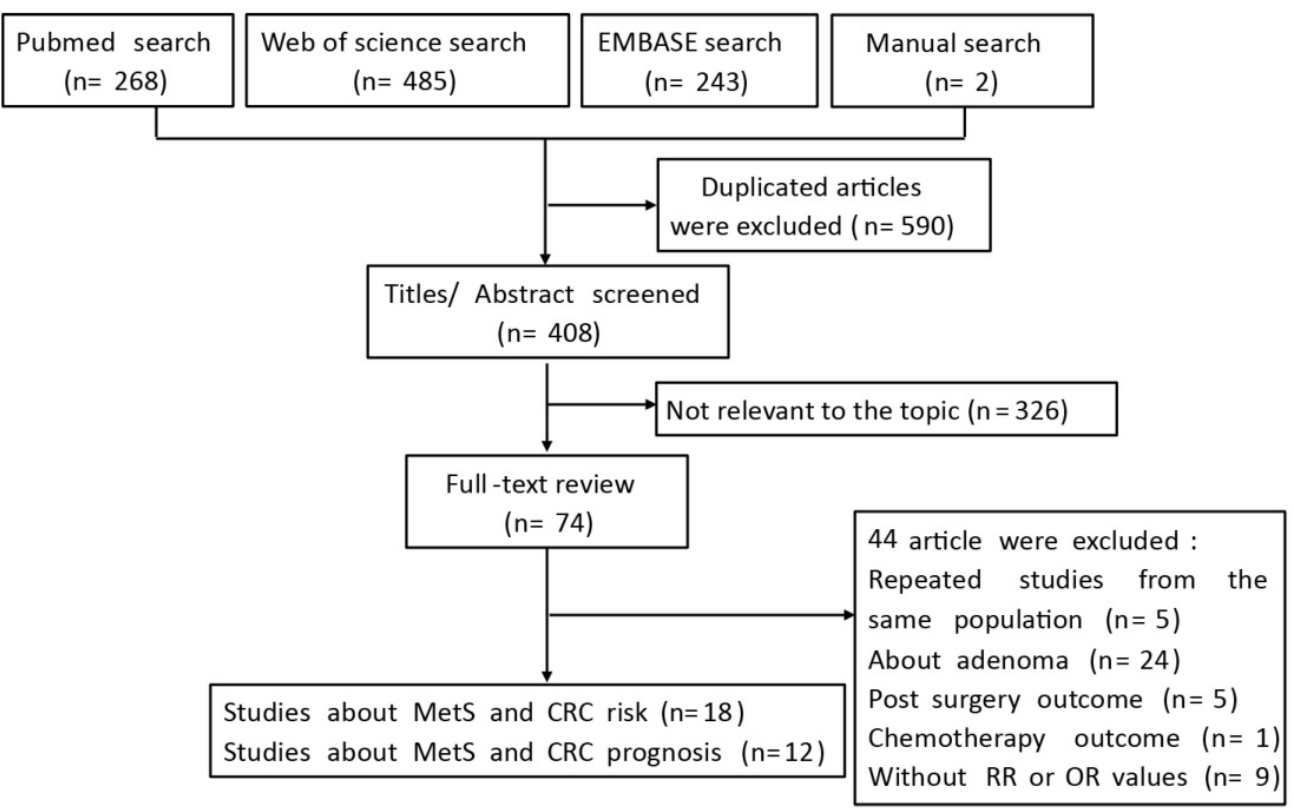

Figure 1. Flow diagram of the systematic literature process. perform these analyses.

\section{Results}

\section{Literature Search and Study Characteristics}

The flow chart of the literature search is presented in Figure 1. After a comprehensive search, a number of 998 citations were identified. Among these, 408 citations remained, after the exclusion of duplicates, and 326 citations were excluded by screening the titles and abstracts, leaving 74 potentially relevant articles for full-text examination. Of these, 44 citations were excluded, because they did not meet the inclusion criteria. Finally, 30 articles were included for data synthesis.

Basic information concerning the eligible studies are listed in Tables 1 and 2. From 2006 to 2019, a total of 10 prospective cohort studies [18-21, 25, 34-38], 6 case-control studies [39-44], one retrospective cohort study [22] and one cross-sectional study [45] were included in the meta-analysis of CRC incidence, and 8 prospective cohort studies [25-27, 46-49], 2 retrospective cohort study $[50,51]$ and 3 case-control studies [52-54] were included in the meta-analysis for CRC mortality. Thirteen studies were conducted in Asia [21, 22, 27, 37, 38, 42, 43, 46, 48, 50-52, 54], eight were performed in Europe [20, 25, 34, 39-41, 44, 45], and the remaining studies were executed in the USA $[18,19,26,35,36,47,49]$. Most of them obeyed the traditional definition from IDF, ATP III or AHA, while some were not. Three studies were limited to males $[19,34,38]$ and two to females $[35,36]$ in the meta-analysis of CRC incidence, and two studies were limited to males $[26,50]$ in the meta-analysis for CRC mortality. As for the article about CRC mortality, four studies investigated stage I-III patients [47, 49, 50, 52] and the remaining studies investigated stage I-IV patients. The level of covariate adjustment in the individual studies differed, most studies adjusted for age, sex, smoking and alcohol. On the basis of the NOS criteria, most studies were classified as highquality, and the remaining studies were classified as moderatequality [20, 22, 40, 42]. 
Table 1. Studies reporting on the association of MetS with CRC incidence

\begin{tabular}{|c|c|c|c|c|c|c|c|c|c|}
\hline $\begin{array}{l}\text { Authors, region, } \\
\text { design, year }\end{array}$ & Sex & $\begin{array}{l}\text { Age (mean } \\
\text { or median } \\
\text { or range) y }\end{array}$ & $\begin{array}{l}\text { Cases } \\
\mathrm{M} / \mathrm{F}\end{array}$ & $\begin{array}{l}\text { Cohort } \\
\text { size or } \\
\text { controls }\end{array}$ & $\begin{array}{l}\text { Follow- } \\
\text { up y }\end{array}$ & Definition of MetS & $\mathrm{RR}, 95 \% \mathrm{CI}$ & Adjustment & QS \\
\hline $\begin{array}{l}\text { Ahmed et al. USA, } \\
\text { Cohort, } 2006\end{array}$ & $\mathrm{M} / \mathrm{F}$ & $45-64$ & $107 / 87$ & $\begin{array}{l}\text { M: } 6630 \\
\text { F: } 7563\end{array}$ & 11.5 & $\begin{array}{l}\geq 3 \text { metabolic } \\
\text { abnormalities }\end{array}$ & $1.39,0.90-2.20$ & $\begin{array}{l}\text { Age, sex, exercise, NSAIDs, } \\
\text { aspirin use, smoking, alcohol }\end{array}$ & 8 \\
\hline $\begin{array}{l}\text { Stürmer et al. USA, } \\
\text { Cohort, } 2006\end{array}$ & $\mathrm{M}$ & 53.8 & 494 & 22046 & 19 & ATP III & $\mathrm{M}, 1.40,0.90-2.10$ & $\begin{array}{l}\text { Age, exercise, smoking, alcohol, } \\
\text { NSAIDs }\end{array}$ & 7 \\
\hline $\begin{array}{l}\text { Bowers et al. } \\
\text { Finland, Cohort, } \\
2006\end{array}$ & $\mathrm{M}$ & 59 & 410 & 28983 & 14.1 & $\begin{array}{l}\geq 3 \text { metabolic } \\
\text { abnormalities }\end{array}$ & $\mathrm{M}, 1.40,1.12-1.74$ & Age, smoking, TC & 8 \\
\hline $\begin{array}{l}\text { Russo et al. Italy, } \\
\text { Cohort, } 2008\end{array}$ & $\mathrm{M} / \mathrm{F}$ & $\geq 40$ & $60 / 61$ & 16677 & 2.7 & $\begin{array}{l}\text { Use of drugs for DM, } \\
\text { hypertension, } \\
\text { hypercholesterolemia }\end{array}$ & $1.08,0.90-1.29$ & NR & 6 \\
\hline $\begin{array}{l}\text { Stocks et al. } \\
\text { Sweden, C/C, } 2008\end{array}$ & $\mathrm{M} / \mathrm{F}$ & $\begin{array}{l}\text { M: } 59.8 \\
\text { F: } 59.4\end{array}$ & $125 / 181$ & $\begin{array}{l}595 \\
\text { controls }\end{array}$ & NR & WHO & $2.57,1.20-5.52$ & $\begin{array}{l}\text { Age, sex, blood sample date, } \\
\text { fasting time }\end{array}$ & 8 \\
\hline $\begin{array}{l}\text { Inoue et al. Japan, } \\
\text { Cohort, } 2009\end{array}$ & $\mathrm{M} / \mathrm{F}$ & $\begin{array}{l}\text { M: } 56.5 \\
\text { F: } 55.5\end{array}$ & $155 / 157$ & $\begin{array}{l}\text { M: } 9548 \\
\text { F: } 18176\end{array}$ & 10.2 & AHA & $\begin{array}{l}\text { M, CC, 1.29, 0.82-2.02 } \\
\text { F, CC, } 1.03,0.65-1.65 \\
\text { M, RC, 0.62, 0.29-1.34 } \\
\text { F, RC, } 0.99,0.51-1.92\end{array}$ & Age, area, smoking, alcohol, TC & 9 \\
\hline $\begin{array}{l}\text { Pelucchi et al. Italy, } \\
\text { C/C, } 2010\end{array}$ & $\mathrm{M} / \mathrm{F}$ & $31-79$ & $1310 / 946$ & $\begin{array}{l}4661 \\
\text { controls }\end{array}$ & NR & IDF & $1.69,1.23-2.33$ & $\begin{array}{l}\text { Age, sex, education, smoking, } \\
\text { alcohol, exercise }\end{array}$ & 6 \\
\hline $\begin{array}{l}\text { Aleksandrova et al. } \\
\text { Europe, C/C, } 2011\end{array}$ & $\mathrm{M} / \mathrm{F}$ & $\begin{array}{l}\mathrm{CC}: 58.8 \\
\mathrm{RC}: 58.1\end{array}$ & $531 / 562$ & $\begin{array}{l}1093 \\
\text { controls }\end{array}$ & 3.7 & ATP III & $\begin{array}{l}\text { CC, } 1.91,1.47-2.42 \\
\text { RC, } 1.45,1.02-2.06\end{array}$ & Dietary consumption & 8 \\
\hline $\begin{array}{l}\text { Li et al. China, } \\
\text { C/C, } 2011\end{array}$ & $\mathrm{M} / \mathrm{F}$ & 59 & $936 / 570$ & $\begin{array}{l}3354 \\
\text { controls }\end{array}$ & NR & IDF & $1.64,1.14-2.49$ & NR & 6 \\
\hline $\begin{array}{l}\text { Stocks et al. } \\
\text { Norway, Austria, } \\
\text { Sweden, Cohort, } \\
2011\end{array}$ & $\mathrm{M} / \mathrm{F}$ & $\begin{array}{l}\text { M: } 43.9 \\
\text { F: } 44.1\end{array}$ & $2834 / 1861$ & $\begin{array}{l}\text { M: } 289866 \\
\text { F: } 288834\end{array}$ & 12 & $\begin{array}{l}\geq 3 \text { metabolic } \\
\text { abnormalities }\end{array}$ & $\begin{array}{l}\mathrm{M}, 1.25,1.18-1.32 \\
\mathrm{~F}, 1.14,1.06-1.22\end{array}$ & $\begin{array}{l}\text { Age, smoking, components of } \\
\text { metabolic abnormalities }\end{array}$ & 8 \\
\hline $\begin{array}{l}\text { Kabat et al. USA, } \\
\text { Cohort, } 2012\end{array}$ & $\mathrm{~F}$ & 64.4 & 81 & 4862 & 12 & ATP III & $\mathrm{F}, 2.15,1.30-3.53$ & $\begin{array}{l}\text { Age, ethnicity, BMI, alcohol, } \\
\text { family history, exercise, } \\
\text { participation trial, treatment }\end{array}$ & 8 \\
\hline $\begin{array}{l}\text { Ulaganathan et al. } \\
\text { Malaysia, C/C, } \\
2012\end{array}$ & $\mathrm{M} / \mathrm{F}$ & 61.5 & $80 / 60$ & $\begin{array}{l}\text { M: } 160 \\
\text { controls } \\
\text { F: } 80 \\
\text { controls }\end{array}$ & NR & IDF & $2.61,1.53-4.47$ & $\begin{array}{l}\text { Age, sex, ethnic, education, } \\
\text { components of MetS, obesity } \\
\text { related biomarkers, energy } \\
\text { intake, exercise, smoking, alcohol }\end{array}$ & 9 \\
\hline $\begin{array}{l}\text { Osaki et al. Japan, } \\
\text { Retrospective } \\
\text { cohort, } 2012\end{array}$ & $\mathrm{M} / \mathrm{F}$ & 58.6 & $98 / 136$ & $\begin{array}{l}\text { M: } 8329 \\
\text { F: } 15386\end{array}$ & 9.1 & IDF & $\begin{array}{l}\text { M, CC, } 1.11,0.55-2.26 \\
\text { M, RC, } 1.84,0.79-4.27 \\
\text { F, CC, } 0.90,0.49-1.65 \\
\text { F, RC, } 1.39,0.60-3.20\end{array}$ & $\begin{array}{l}\text { Age, smoking, alcohol, presence } \\
\text { of MetS or pre-MetS of each } \\
\text { definition }\end{array}$ & 6 \\
\hline $\begin{array}{l}\text { Kontou et al. } \\
\text { Greece, C/C, } 2012\end{array}$ & $\mathrm{M} / \mathrm{F}$ & 62 & $146 / 93$ & $\begin{array}{l}250 \\
\text { controls }\end{array}$ & NR & ATP III & $1.66,1.02-2.69$ & $\begin{array}{l}\text { Age, sex, BMI, family history, } \\
\text { exercise, smoking }\end{array}$ & 8 \\
\hline $\begin{array}{l}\text { Liang et al. USA, } \\
\text { Cohort, } 2017\end{array}$ & $\mathrm{~F}$ & 66.7 & 114 & 5068 & 14.3 & ATP III & $\mathrm{F}, 1.49,1.02-2.18$ & $\begin{array}{l}\text { Age, ethnicity, smoking, alcohol, } \\
\text { exercise, energy intake, dietary } \\
\text { fiber, calories percent, family } \\
\text { history, NSAIDs, treatment }\end{array}$ & 9 \\
\hline $\begin{array}{l}\text { Choi et al. Korea, } \\
\text { Cohort, } 2018\end{array}$ & $\mathrm{M} / \mathrm{F}$ & 54 & 63045 & 6296903 & 5.3 & IDF & $1.22,1.20-1.24$ & $\begin{array}{l}\text { Age, sex, smoking, alcohol, } \\
\text { exercise }\end{array}$ & 9 \\
\hline $\begin{array}{l}\text { Milano et al. Italy, } \\
\text { Cross-sectional, } \\
2019\end{array}$ & $\mathrm{M} / \mathrm{F}$ & 61 & 213 & 5707 & NR & AHA & $1.92,1.42-2.58$ & $\begin{array}{l}\text { Age, sex, exercise, component of } \\
\text { MetS }\end{array}$ & 7 \\
\hline $\begin{array}{l}\text { Li et al. China, } \\
\text { Cohort, } 2019\end{array}$ & $\mathrm{M}$ & 51.2 & 394 & 104333 & 8.9 & AHA & $1.22,0.97-1.53$ & $\begin{array}{l}\text { Age, education, income, } \\
\text { smoking, alcohol, sitting time }\end{array}$ & 9 \\
\hline
\end{tabular}

Table 2. Studies reporting on the association of MetS with CRC mortality

\begin{tabular}{|c|c|c|c|c|c|c|c|c|c|c|}
\hline $\begin{array}{l}\text { Authors, region, } \\
\text { design, year }\end{array}$ & Sex & $\begin{array}{l}\text { Age (mean } \\
\text { or median } \\
\text { or range) y }\end{array}$ & $\begin{array}{l}\text { Cohort } \\
\text { size or } \\
\text { controls }\end{array}$ & $\begin{array}{l}\text { Follow-up } \\
\text { period, } \\
\text { month }\end{array}$ & $\begin{array}{l}\text { Definition of } \\
\text { MetS }\end{array}$ & RR, 95\% CI & Adjustment & Stage & Survival & QS \\
\hline $\begin{array}{l}\text { Shen et al. China, } \\
\text { Cohort, } 2010\end{array}$ & $\mathrm{M} / \mathrm{F}$ & 64.1 & 507 & 45.1 & $\begin{array}{l}\geq 3 \text { metabolic } \\
\text { abnormalities }\end{array}$ & $\begin{array}{l}\text { CC, } 1.63,1.04-2.57 \\
\text { RC, } 1.94,1.08-3.50\end{array}$ & NR & I-IV & CSS & 7 \\
\hline $\begin{array}{l}\text { Matthews et al. } \\
\text { USA, Cohort, } \\
2010\end{array}$ & M & 47.2 & 33230 & 14.4 & ATPIII & $1.71,0.97-3.02$ & $\begin{array}{l}\text { Age, examination year, height, smoking, } \\
\text { alcohol, family history, treadmill test } \\
\text { duration }\end{array}$ & I-IV & OS & 9 \\
\hline $\begin{array}{l}\text { Stocks et al. } \\
\text { Europe, Cohort, } \\
2011\end{array}$ & $\mathrm{M} / \mathrm{F}$ & $\begin{array}{l}\mathrm{M}, 43.9 \\
\mathrm{~F}, 44.1\end{array}$ & $\begin{array}{l}\text { M: } 2761 \\
\text { F: } 1815\end{array}$ & 12 & $\begin{array}{l}\geq 3 \text { metabolic } \\
\text { abnormalities }\end{array}$ & $\begin{array}{l}\mathrm{M}, 1.25,1.18-1.32 \\
\mathrm{~F}, 1.14,1.06-1.22\end{array}$ & $\begin{array}{l}\text { Age, smoking, components of metabolic } \\
\text { abnormalities }\end{array}$ & I-IV & OS & 8 \\
\hline $\begin{array}{l}\text { Yang et al. China, } \\
\text { Cohort, } 2013\end{array}$ & $\mathrm{M} / \mathrm{F}$ & 77.1 & 36079 & 72 & ATPIII & $0.98,0.93-1.02$ & $\begin{array}{l}\text { Age, sex, race, marital status, education, } \\
\text { income, comorbidity, year of diagnosis, }\end{array}$ & I-IV & OS & 9 \\
\hline
\end{tabular}




\begin{tabular}{|c|c|c|c|c|c|c|c|c|c|c|}
\hline & & & & & & & stage, grade & & & \\
\hline $\begin{array}{l}\text { Ahmadi et al. } \\
\text { Iran, C/C, } 2015\end{array}$ & $\mathrm{M} / \mathrm{F}$ & 54 & 1127 & 25 & NR & $0.95,0.52-1.50$ & $\begin{array}{l}\text { Age, sex, smoking, tumor size, } \\
\text { histological type, differentiation, stage, } \\
\text { family history, education, alcohol, } \\
\text { marital status }\end{array}$ & I-IV & OS & 9 \\
\hline $\begin{array}{l}\text { You et al. China, } \\
\text { C/C, } 2015\end{array}$ & $\mathrm{M} / \mathrm{F}$ & 67 & 1069 & 59.6 & CDS & $0.79,0.59-1.06$ & $\begin{array}{l}\text { Age, sex, stage, differentiation, HDL, } \\
\text { uric acid, carcinoembryonie antigen }\end{array}$ & I-III & OS & 9 \\
\hline $\begin{array}{l}\text { Cespedes et al. } \\
\text { USA, Cohort, } \\
2016\end{array}$ & $\mathrm{M} / \mathrm{F}$ & 64 & 2446 & 72 & AHA & $1.23,1.03-1.56$ & $\begin{array}{l}\text { Age, race, sex, smoking, stage, grade, } \\
\text { chemotherapy, radiation, site, } \\
\text { sex-specific tertile of muscle tissue at } \\
\text { diagnosis }\end{array}$ & I-III & OS & 9 \\
\hline $\begin{array}{l}\text { Peng et al. China, } \\
\text { Cohort, } 2016\end{array}$ & $\mathrm{M} / \mathrm{F}$ & 56.4 & 1318 & 58.6 & CDS & $2.98,2.40-3.69$ & $\begin{array}{l}\text { Age, sex, smoking, alcohol, family } \\
\text { history, year of diagnosis, cancer site, } \\
\text { stage }\end{array}$ & I-IV & CSS & 9 \\
\hline $\begin{array}{l}\text { You et al. China, } \\
\text { C/C, } 2017\end{array}$ & $\mathrm{M} / \mathrm{F}$ & 65.2 & 1163 & 71.2 & CDS & $0.93,0.83-1.05$ & $\begin{array}{l}\text { Age, sex, platelet to lymphocyte ratio, } \\
\text { stage }\end{array}$ & I-IV & OS & 8 \\
\hline $\begin{array}{l}\text { Chen et al. China, } \\
\text { R/C, } 2018\end{array}$ & $\mathrm{M}$ & 50.9 & 838 & 40.6 & CDS & $1.13,1.06-1.48$ & NR & I-III & OS & 8 \\
\hline $\begin{array}{l}\text { Croft et al. } \\
\text { Canada, Cohort, } \\
2019\end{array}$ & $\mathrm{M} / \mathrm{F}$ & 68.9 & 142 & 65.3 & NR & $1.09,0.27-4.30$ & NR & I-III & OS & 8 \\
\hline $\begin{array}{l}\text { Mafiana et al. } \\
\text { Arab, R/C, } 2019\end{array}$ & $\mathrm{M} / \mathrm{F}$ & 55 & 301 & NA & AHA & $1.01,0.64-1.59$ & $\begin{array}{l}\text { Age, sex, stage, differentiation, cancer } \\
\text { treatment, alcohol, smoking }\end{array}$ & I-IV & CSS & 9 \\
\hline
\end{tabular}

\begin{tabular}{|c|c|c|c|}
\hline Study ID & & $\mathrm{ES}(95 \% \mathrm{Cl})$ & Weight $\%$ \\
\hline Ahmed et al (2006) & 1 & $1.39(0.90,2.20)$ & 1.21 \\
\hline Stürmer et al (2006) & $\frac{1}{1}$ & $1.40(0.90,2.10)$ & 1.41 \\
\hline Bowers et al (2006) & $\rightarrow$ & $1.40(1.12,1.74)$ & 4.50 \\
\hline Russo et al (2008) & $\rightarrow$ & $1.08(0.90,1.29)$ & 8.72 \\
\hline Stocks et al (2008) & + & $2.57(1.20,5.52)$ & 0.12 \\
\hline Inoue et al (2009) (M, CC) & 1 & $1.29(0.82,2.02)$ & 1.41 \\
\hline Inoue et al (2009) (F, CC) & H' & $0.62(0.29,1.32)$ & 1.87 \\
\hline Inoue et al (2009) (M, RC) & & $1.03(0.65,1.65)$ & 1.98 \\
\hline Inoue et al (2009) $(F, R C)$ & & $0.99(0.51,1.92)$ & 1.04 \\
\hline Pelucchi et al (2010) & & $1.69(1.23,2.33)$ & 1.66 \\
\hline Aleksandrova et al (2011) (CC) & $\leftarrow$ & $1.91(1.47,2.42)$ & 2.17 \\
\hline Aleksandrova et al (2011) (RC) & & $1.45(1.02,2.06)$ & 1.84 \\
\hline Li et al (2011) & & $1.64(1.14,2.49)$ & 1.13 \\
\hline Stocks et al (2011) (M) & $\bullet$ & $1.25(1.18,1.32)$ & 18.67 \\
\hline Stocks et al (2011) (F) & - & $1.14(1.06,1.22)$ & 17.75 \\
\hline Kabat et al (2012) & & $2.15(1.30,3.53)$ & 0.43 \\
\hline Ulaganathan et al (2012) & ' & $2.61(1.53,4.47)$ & 0.25 \\
\hline Osaki et al (2012) (M, CC) & $\frac{1}{6}$ & $1.11(0.55,2.26)$ & 0.72 \\
\hline Osaki et al (2012) (M, RC) & & $1.84(0.79,4.27)$ & 0.18 \\
\hline Osaki et al (2012) (F, CC) & + & $0.90(0.49,1.65)$ & 1.50 \\
\hline Osaki et al (2012) (F, RC) & 7 & $1.39(0.60,3.20)$ & 0.32 \\
\hline Kontou et al (2012) & 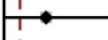 & $1.66(1.02,2.69)$ & 0.75 \\
\hline Liang et al (2017) & 1 & $1.49(1.02,2.18)$ & 1.50 \\
\hline Choi et al (2018) & - & $1.22(1.20,1.24)$ & 22.10 \\
\hline Milano et al (2019) & $1 \rightarrow$ & $1.92(1.42,2.58)$ & 1.50 \\
\hline Li et al (2019) & + & $1.22(0.97,1.53)$ & 5.28 \\
\hline Overall $(I-$ squared $=44.1 \%, p=0.009)$ & $\theta$ & $1.25(1.18,1.32)$ & 100.00 \\
\hline NOTE: Weights are from random effects analysis & & & \\
\hline
\end{tabular}

Figure 2. Forest plot to quantify the association between MetS and CRC incidence. ES, effect size; $95 \% \mathrm{Cl}, 95 \%$ confidence interval; $\mathrm{M}$, male; F, female; CC, colon cancer; RC, rectal cancer.

\section{Meta-Analysis for association between MetS and CRC}

In the meta-analysis combining results of the included studies, MetS was associated with CRC risk
(Summary $\mathrm{RR}=1.25 ; 95 \%$ CI: 1.18-1.32), with a between-study heterogeneity $(\mathrm{Q}(d f=25)=44.7$, $p$-value $=0.009 ; I^{2}=44.1 \%$ ) (Figure 2). In overall analysis, the presence of MetS was associated with a $15 \%$ increased mortality risk in CRC (Summary RR = 
1.15; 95\% CI: 1.02-1.28), and this association was obsessed by significant between-study heterogeneity $\left(\mathrm{Q}(d f=13)=96.56, p\right.$-value $\left.=0.000 ; I^{2}=86.5 \%\right)($ Figure 3).

\section{Subgroup Analysis}

In terms of total CRC incidence (Figure 4A), a RR of 1.28 (95\% CI: 1.16-1.39) was found in male, with a between-study heterogeneity $\left(p\right.$-value $=0.009 ; I^{2}=$ $60.8 \%)$. In articles reporting incidence of CRC in female patients with MetS, a RR of 1.21 (95 \% CI: 1.13-1.30) was found. However, no significant heterogeneity among the studies was found ( $p$-value $\left.=0.212 ; I^{2}=20.7 \%\right)$. Studies were divided according to average age of included patients, the older people with MetS suffer a higher risk of CRC. Studies were divided according to cancer site, and the colon cancer group showed an association between MetS and cancer risk (Pooled RR $=1.23$; 95\% CI: 1.10-1.37; $p$-value $\left.=0.029 ; I^{2}=46.4 \%\right)$, and also there was a significant correlation in the rectal cancer subgroup (Pooled RR $=1.18 ; 95 \%$ CI: 1.10-1.26; $p$-value $=0.307 ; I^{2}$ $=13.7 \%$ ). Studies were divided according to study type, both cohort and case-control study showed a tight association between MetS and CRC risk (Pooled $\mathrm{RR}=1.22$; 95\% CI: 1.20-1.24; and Pooled RR = 1.73; 95\% CI: 1.47-1.98, respectively), and with no significant heterogeneity ( $p$-value $=0.283, I^{2}=14.3 \%$; and $p$-value $=0.712, I^{2}=0 \%$, respectively). As our data showed, studies conducted in USA and Asia, with publication year $\geq 2012$, and with MetS defined by ATP III or IDF showed a more prominent association between MetS and CRC incidence and a good homogeneity.

In terms of CRC mortality (Figure 4B), by survival outcome, significance was found in studies investigating cancer-specific survival (CSS) (Pooled $\mathrm{RR}=1.72 ; 95 \% \mathrm{CI}: 1.03-2.42 ; p$-value $=0.000 ; I^{2}=$ $84.6 \%$ ), but not in the overall survival (OS) (Pooled RR $=1.07 ; 95 \%$ CI: 0.96-1.18; $p$-value $=0.000 ; I^{2}=85.2 \%$. Studies were analyzed according to average age, and we found that the older people with MetS suffer a higher risk of mortality. A RR of 1.24 (95\% CI: 1.18-1.39) was found in male, with a between-study heterogeneity $\left(p\right.$-value $\left.=0.433 ; I^{2}=0 \%\right)$, however, only two articles reported specific mortality in female, and a RR of 0.98 (95\% CI: 0.64-1.32) was found with a significant heterogeneity ( $p$-value $=0.006 ; I^{2}=86.9 \%$ ). By study design, the association between MetS and CRC mortality was statistically significant in cohort studies (Pooled RR $=1.26 ; 95 \%$ CI: 1.10-1.41). Since most studies were conducted in Chinese, we split the studies into Chinese by ethnicity, and found that there was no significant correlation between MetS and CRC mortality in Chinese. Articles with MetS defined by $\geq 3$ metabolic abnormalities showed a more prominent association between MetS and CRC incidence. Grouping studies according to TNM stage, risk magnitude did not differ between the two groups.

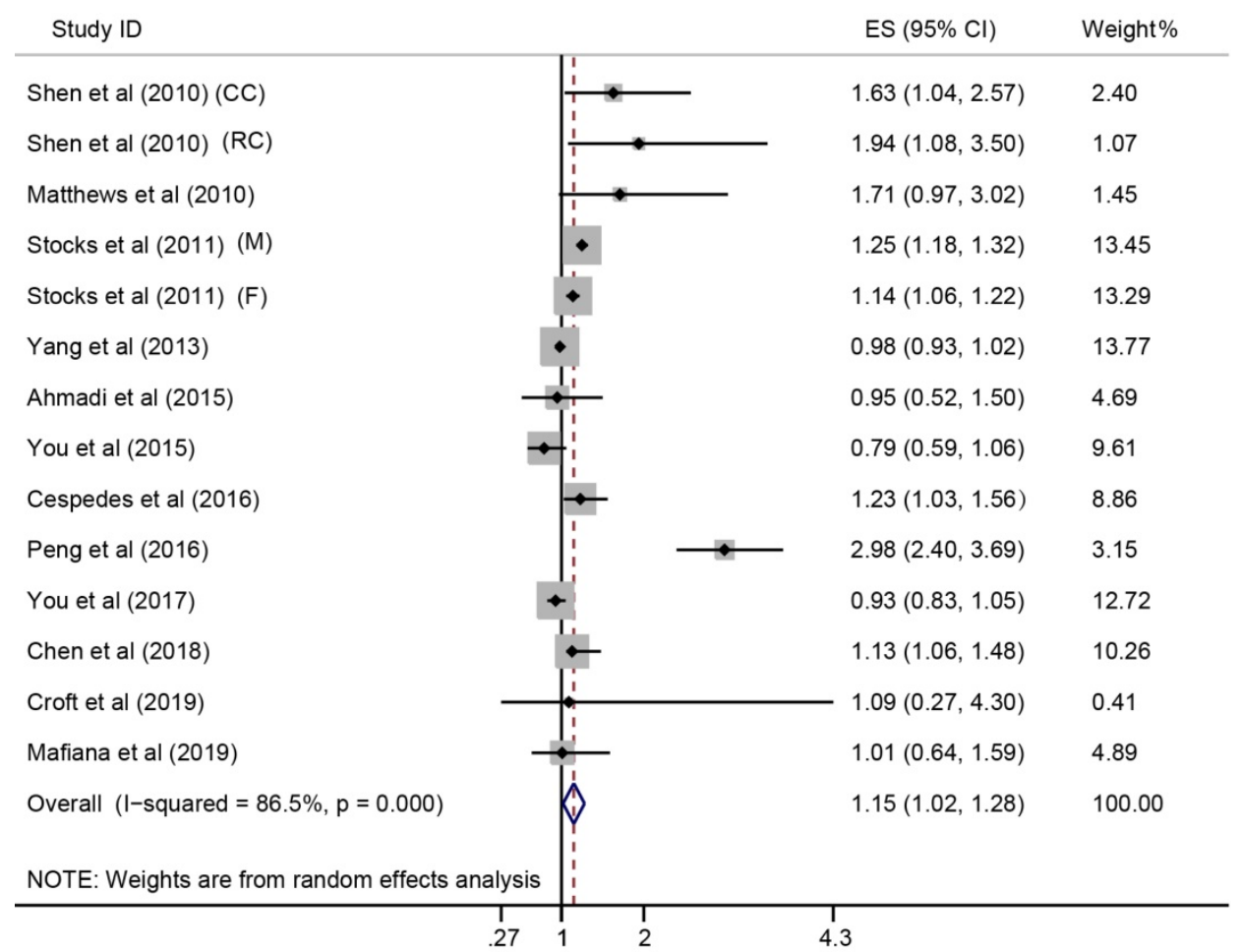

Figure 3. Forest plot for the association between MetS and CRC survival. ES, effect size; $95 \% \mathrm{Cl}, 95 \%$ confidence interval; $\mathrm{M}$, male; $\mathrm{F}$, female; CC, colon cancer; RC, rectal cancer. 


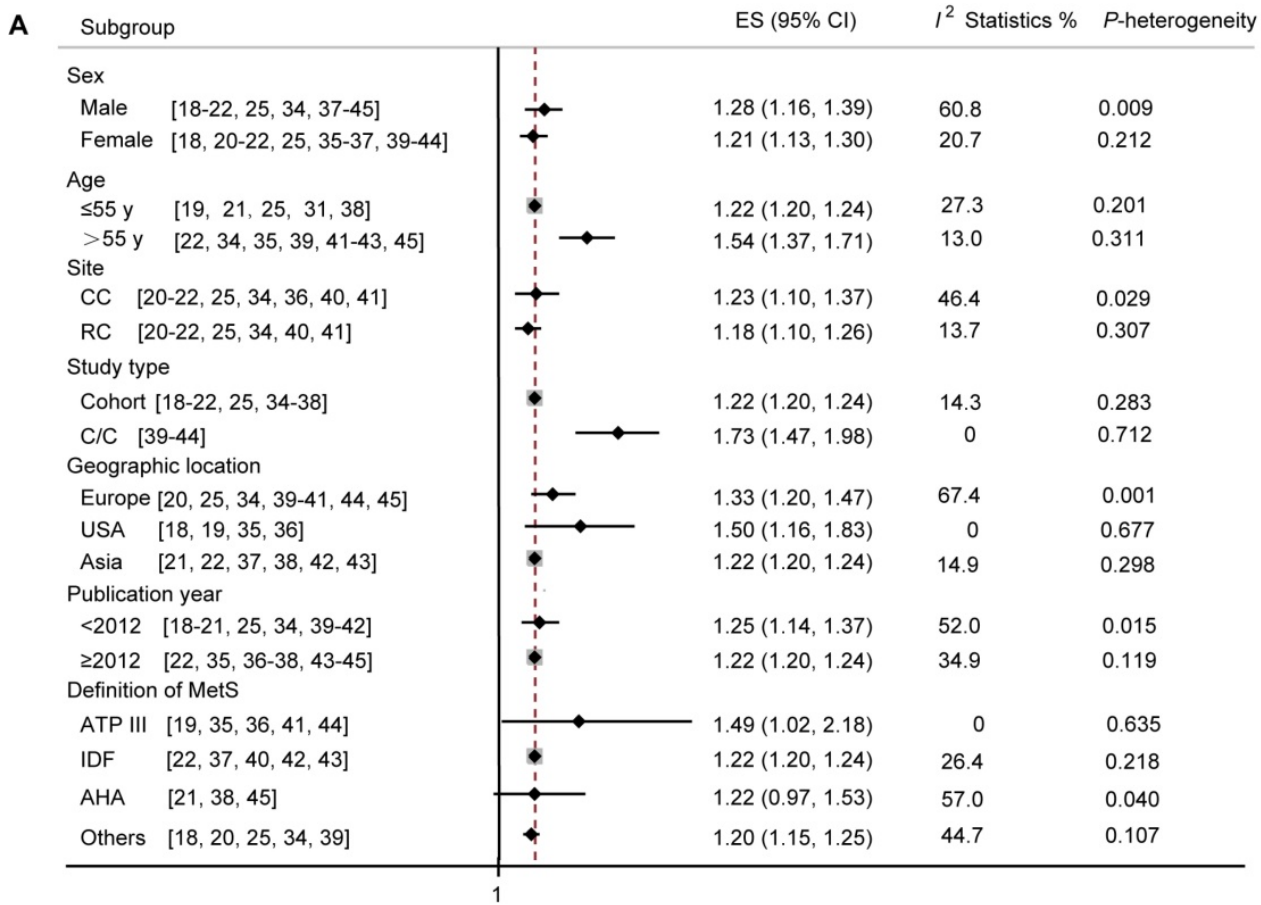

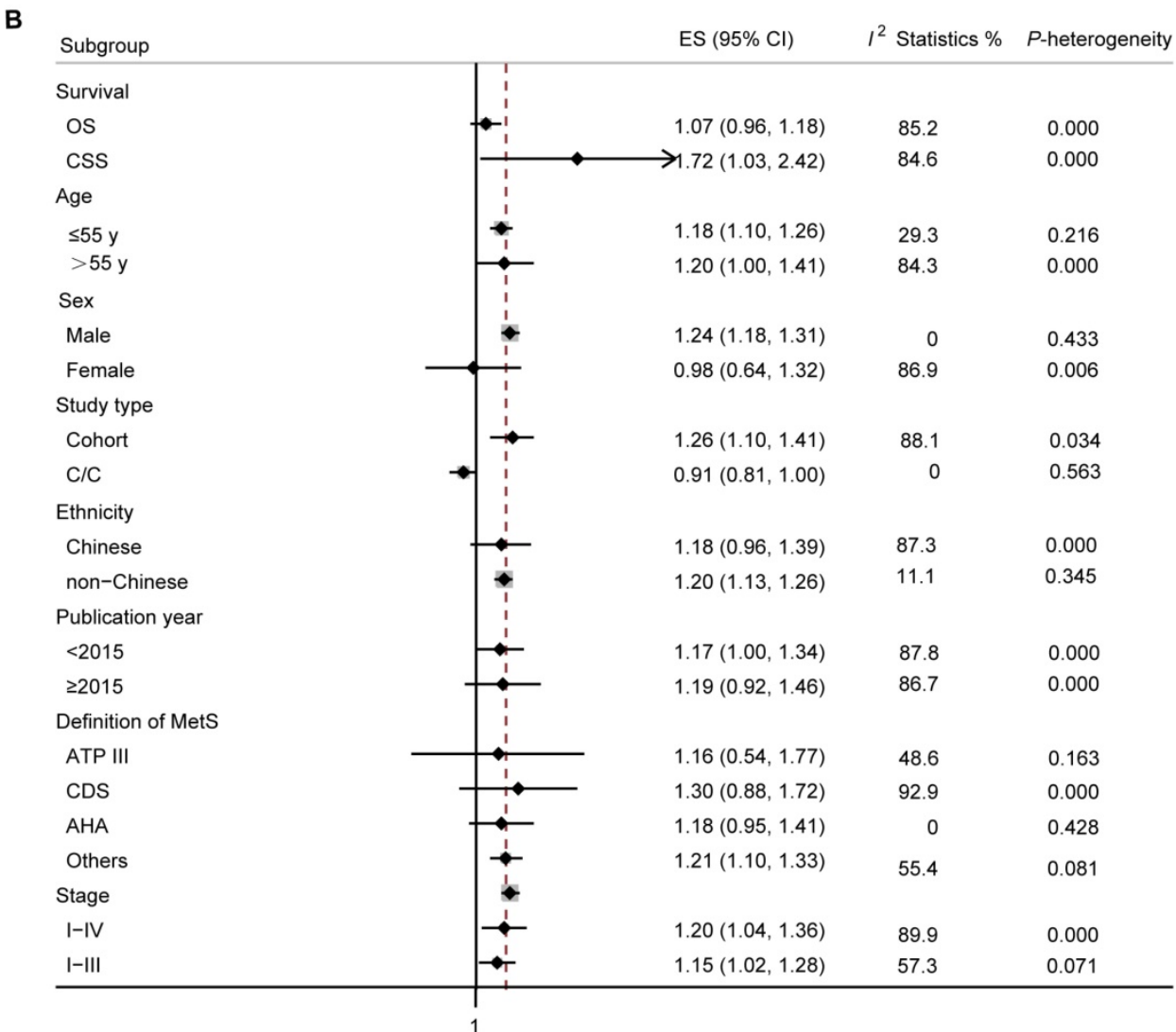

Figure 4. Subgroup analyses for cancer incidence and survival in patients with CRC. (A) Subgroup analyses for the association between MetS and CRC incidence. (B) Subgroup analyses for the association between MetS and survival in patients with CRC. ES, effect size; $95 \%$ Cl, $95 \%$ confidence interval; IDF: International Diabetes Federation; AHA: American Heart Association; ATP III: Adult Treatment Panel III; CDS: Chinese Diabetes Society; OS: overall survival; CSS: cancer-specific survival.

\section{Association between individual components of MetS and CRC}

The influence of any single component of MetS on CRC incidence and mortality was summarized in
Table 3 and the detailed information was presented in Table S1 and S2. In terms of CRC incidence, both obesity and dysglycemia (high fasting or postprandial glucose, or reported diabetes) are significant risk factors for the incidence of CRC regardless of the sex. 
Interestingly, both hypertension and hyperlipoidemia were risk factors for the incidence in male but not in female. In terms of CRC mortality, only dysglycemia was a significant risk factor for the mortality of CRC, with significant heterogeneity between studies. Obesity tended to be associated with an increased mortality risk.

Table 3. Association between individual components of MetS and CRC

\begin{tabular}{lllll}
\hline Stratification factor & Sex & ES, 95\% CI & Heterogeneity, $I^{2}$ & $p$ value \\
\hline Incidence & & & & \\
Obesity & $\mathrm{M}+\mathrm{F}$ & $1.11,1.06-1.16$ & $41.5 \%$ & 0.034 \\
& $\mathrm{M}$ & $1.12,1.09-1.16$ & $39.2 \%$ & 0.117 \\
Hypertension & $\mathrm{F}$ & $1.10,1.00-1.20$ & $56.4 \%$ & 0.025 \\
& $\mathrm{M}+\mathrm{F}$ & $1.04,1.01-1.06$ & $37.1 \%$ & 0.058 \\
& $\mathrm{M}$ & $1.09,1.06-1.13$ & $0.0 \%$ & 0.950 \\
Dysglycemia & $\mathrm{F}$ & $0.97,0.84-1.10$ & $75.8 \%$ & 0.000 \\
& $\mathrm{M}+\mathrm{F}$ & $1.14,1.11-1.17$ & $9.2 \%$ & 0.345 \\
& $\mathrm{M}$ & $1.16,1.11-1.20$ & $22.9 \%$ & 0.247 \\
Hyperlipoidemia & $\mathrm{F}$ & $1.21,1.17-1.26$ & $0.0 \%$ & 0.782 \\
& $\mathrm{M}+\mathrm{F}$ & $1.00,0.94-1.06$ & $60.1 \%$ & 0.001 \\
& $\mathrm{M}$ & $1.10,1.05-1.15$ & $48.9 \%$ & 0.057 \\
Low HDL-C & $\mathrm{F}$ & $1.03,0.99-1.07$ & $41.7 \%$ & 0.100 \\
Mortality & $\mathrm{M}+\mathrm{F}$ & $1.04,0.87-1.21$ & $57.3 \%$ & 0.016 \\
Obesity & & & & \\
Hypertension & $\mathrm{M}+\mathrm{F}$ & $1.04,0.97-1.12$ & $59.7 \%$ & 0.015 \\
Dysglycemia & $\mathrm{M}+\mathrm{F}$ & $1.01,0.91-1.11$ & $66.0 \%$ & 0.003 \\
Hyperlipoidemia & $\mathrm{M}+\mathrm{F}$ & $1.10,1.01-1.20$ & $59.1 \%$ & 0.007 \\
\hline
\end{tabular}

ES: effect size; 95\% CI: 95\% confidence interval; M: male; F: female.

\section{Sensitivity analysis and publication bias}

Sensitivity analyses were conducted to examine the stability of the estimates for the accociation between MetS and incidence and mortality of CRC (Figure S1). The sensitivity analysis showed the summary RRs were not markedly changed by any individual study, indicating no significant influence of single study on the results. Non-significant publication bias was found for either of incidence (Kendall's tau $=-0.02, p=1.00$ ) or survival (Kendall's tau $=0.71, p=0.48$ ).

\section{Discussion}

This meta-analysis focused on the association of MetS and its components with the incidence and progression of colorectal cancer, involving 18 studies with incidence and 12 studies with survival outcomes, respectively. The results from this meta-analysis indicated that MetS is associated with an increased risk of CRC incidence and mortality. We observed $25 \%$ increased cancer incidence, and 15\% increased cancer mortality in patients with MetS. In a metaanalysis [55], which discussed the correlation of MetS with digestive tract cancer, no significant association was observed between MetS and CRC mortality. Another meta-analysis on the relationship between
MetS and CRC incidence and mortality was performed in 2013 [28]. In this mentioned metaanalysis, MetS is associated with an increased risk of CRC incidence and mortality both in male and female. However, in this current study, we found no significant correlation between MetS and CRC mortality in female.

In subgroup analysis, the risk estimates of CRC incidence changed little depending on sex, age, cancer site (colon and rectum), type of studies (cohort vs non cohort), ethnicity (Europe, USA, Asia), publication year, or definition of MetS. According to previous studies, MetS may increase the risk of CRC in male rather than female $[18,20]$. In contrast, according to our meta-analysis, the risk estimates of CRC incidence changed little depending on sex. People with age over 55 years old may suffer more from MetS. In addition, the correlation may also differ because of cancer site [23]. However, in this current meta-analysis, there is no significance between the two different cancer sites. A study conducted in South Asians, indicated that definition of MetS by the IDF is the most sensitive in predicting the risk of $\mathrm{CRC}$, compared to MetS as defined by the WHO and ATP III [56]. According to our meta-analysis, MetS increased the risk of CRC as defined either IDF or ATPIII, other than defined as AHA.

As for CRC mortality, further stratified analysis indicated statistical significance in studies with assessing cancer-specific survival outcome, in male, a cohort design, ethnicity of non-Chinese or with definition of Met $S$ as $\geq 3$ metabolic abnormalities. The risk estimates of CRC mortality changed little depending on age; however, older people with MetS may suffer a higher mortality of CRC. According to our meta-analysis, MetS has no effect on CRC mortality in female. However, an obvious heterogeneity was observed and might decrease the reliability. So, more studies are needed to further clarify this. Since most of the studies were conducted in China, the associations in Europe and USA should be further considered. In addition, most studies identified the overall survival of CRC with MetS, however, the data of cancer-specific survival outcome should be enriched.

Metabolic syndrome (MetS) is a cluster of metabolic risk factors that includes abdominal obesity, hypertension, hyperglycemia, and dyslipidemia, mainly high serum triglyceride and low serum high-density lipoprotein [1]. In our metaanalysis, obesity and hyperglycemia are risk factors of CRC incidence in both male and female, which were in consistence with the previous meta-analysis [28]. Hypertension and hyperlipoidemia were also indicated risk factors for CRC incidence, especially in 
male. Since the numbers were too little for statistical analysis, we didn't discuss the difference of sex in low HDL-cholesterol. Experimental studies showed that HDL-cholesterol might promote tumorigenesis through regulation of apoptosis or its influence on cell cycle entry [57], which might explain the role of low HDL-cholesterol in increasing the risk of CRC. Since almost no studies showed the relationship between MetS and CRC mortality in different sex, so we didn't discuss the association according to sex. As our data showed that only dysglycemia is the risk factor for both CRC incidence and mortality. Dysglycemia (high fasting or postprandial glucose, or reported diabetes) may act as carcinogenic agent through promoting the epithelial mesenchymal transition phenomenon [58] and promoting cancer cell proliferation [59]. Uptaking of high glucose by cancer cells is associated with advanced grading, greater metastatic potential and cancer chemotherapy resistance [60].

There are potential limitations existing in our study which should be considered. Significant heterogeneity was observed between the studies. Important confounders were not always fully controlled for, which might result in some overestimation of effects due to residual confounding. Studies included used different factors and cut-off points, which complicate comparisons between studies.

In conclusion, MetS is associated with an increased risk of CRC incidence and cancer-specific mortality, but not overall mortality. As for age, the older MetS patients (over 55 years old) are with an increased risk of CRC incidence and mortality. In addition, MetS may increase the CRC mortality in male rather than in female. Moreover, MetS increases the CRC mortality in non-Chinese rather than in Chinese. However, since most of the studies on CRC mortality were conducted in Chinese, further studies are needed to clarify this connection. Among the single components of the syndrome, dysglycemia was the only factor that increased the risk of incidence and mortality of CRC. The pathophysiological mechanisms between MetS and CRC should be further clarified. Our study fully clarified the association of MetS and its components with both CRC incidence and survival. Especially, our results will provide reference in the strategies of CRC prevention and managements.

\section{Abbreviations}

MetS: Metabolic syndrome; CRC: Colorectal cancer; CIs: Confidences Intervals; NOS: Newcastle Ottawa Scale; RCTs: randomized controlled trials; PRISM: preferred reporting items for systematic reviews and meta-analyses; BMI: Body mass index;
RR: relative ratio; M: male; F: female; N.R: not reported; NSAIDs: non-steroidal anti-inflammatory drugs; TC: total cholesterol; DM: diabetic mellitus; AHA: American Heart Association; ATP III: Adult Treatment Panel III; C/C: Case-control; IDF: International Diabetes Federation; WHO: World Health Organization; HDL: high-density lipoprotein; R/C: Retrospective cohort; CDS: Chinese Diabetes Society; OS: overall survival; CSS: cancer-specific survival; ES: effect size.

\section{Supplementary Material}

Supplementary figures and tables. http://www.ijbs.com/v17p0487s1.pdf

\section{Acknowledgements}

\section{Funding}

This work was supported by the National Natural Science Foundation of China (no. 81900740), the Scientific Research Funding of Tianjin Medical University Chu Hsien-I Memorial Hospital (2018RC02), the Tianjin Union Medical Center Project (no. 2020YJ002) and the Tianjin Health Commission Science and Technology Project (no. QN20025).

\section{Authors' contributions}

Fei Han and Guanghai $\mathrm{Wu}$ performed the statistical analyses and wrote the paper. Shuai Zhang and Judong Zhang have screened the literature and selected papers for inclusion in the review. Yongjie Zhao contributed to data extraction and study designing. Jing $\mathrm{Xu}$ revised this paper and designed the study. All authors contributed to the development of this manuscript and read and approved the final version.

\section{Competing Interests}

The authors have declared that no competing interest exists.

\section{References}

1. Eckel RH, Grundy SM, Zimmet PZ. The metabolic syndrome. Lancet. 2005; 365: 1415-28.

2. Grundy SM. Metabolic syndrome pandemic. Arteriosclerosis, thrombosis, and vascular biology. 2008; 28: 629-36

3. Martinez MA, Puig JG, Mora M, Aragon R, O'Dogherty P, Anton JL, et al. Metabolic syndrome: prevalence, associated factors, and C-reactive protein: the MADRIC (MADrid RIesgo Cardiovascular) Study. Metabolism: clinical and experimental. 2008; 57: 1232-40.

4. Loucks EB, Rehkopf DH, Thurston RC, Kawachi I. Socioeconomic disparities in metabolic syndrome differ by gender: evidence from NHANES III. Annals of epidemiology. 2007; 17: 19-26.

5. Marcotte-Chenard A, Deshayes TA, Ghachem A, Brochu M. Prevalence of the metabolic syndrome between 1999 and 2014 in the United States adult population and the impact of the 2007-2008 recession: an NHANES study. Applied physiology, nutrition, and metabolism = Physiologie appliquee, nutrition et metabolisme. 2019; 44: 861-8.

6. Esposito K, Chiodini P, Colao A, Lenzi A, Giugliano D. Metabolic syndrome and risk of cancer: a systematic review and meta-analysis. Diabetes Care. 2012; 35: 2402-11 
7. Bellastella G, Scappaticcio L, Esposito K, Giugliano D, Maiorino MI. Metabolic syndrome and cancer: "The common soil hypothesis". Diabetes research and clinical practice. 2018; 143: 389-97.

8. Bizzarri C, Bottaro G, Pinto RM, Cappa M. Metabolic syndrome and diabetes mellitus in childhood cancer survivors. Pediatric endocrinology reviews : PER. 2014; 11: 365-73.

9. Torre LA, Bray F, Siegel RL, Ferlay J, Lortet-Tieulent J, Jemal A. Global cancer statistics, 2012. CA: a cancer journal for clinicians. 2015; 65: 87-108.

10. Arnold M, Sierra MS, Laversanne M, Soerjomataram I, Jemal A, Bray F. Global patterns and trends in colorectal cancer incidence and mortality. Gut. 2017; 66: 683-91.

11. Fernandez-Villa T, Alvarez-Alvarez L, Rubin-Garcia M, Obon-Santacana M, Moreno V. The role of dietary patterns in colorectal cancer: a 2019 update. Expert review of gastroenterology \& hepatology. 2020; p: 1-10.

12. Le Marchand L, Wilkens LR, Castelfranco AM, Monroe KR, Kristal BS, Cheng I, et al. Circulating Biomarker Score for Visceral Fat and Risks of Incident Colorectal and Postmenopausal Breast Cancer: The Multiethnic Cohort Adiposity Phenotype Study. Cancer epidemiology, biomarkers \& prevention. 2020; 29(5):966-973.

13. Nam S, Choi YJ, Kim DW, Park EC, Kang JG. Risk Factors for Colorectal Cancer in Korea: A Population-Based Retrospective Cohort Study. Annals of coloproctology. 2019; 35: 347-56

14. Xia Z, Su YR, Petersen P, Qi L, Kim AE, Figueiredo JC, et al. Functional informed genome-wide interaction analysis of body mass index, diabetes and colorectal cancer risk. Cancer medicine. 2020; 9: 3563-73.

15. Lee J, Lee KS, Kim H, Jeong H, Choi MJ, Yoo HW, et al. The relationship between metabolic syndrome and the incidence of colorectal cancer. Environ Health Prev Med. 2020; 25: 6.

16. Chen H, Zheng X, Zong X, Li Z, Li N, Hur J, et al. Metabolic syndrome, metabolic comorbid conditions and risk of early-onset colorectal cancer. Gut. 2020.

17. Elangovan A, Skeans J, Landsman M, Ali SMJ, Elangovan AG, Kaelber DC, et al. Colorectal Cancer, Age, and Obesity-Related Comorbidities: A Large Database Study. Dig Dis Sci. 2020

18. Ahmed RL, Schmitz KH, Anderson KE, Rosamond WD, Folsom AR. The metabolic syndrome and risk of incident colorectal cancer. Cancer. 2006; 107: 28-36.

19. Sturmer T, Buring JE, Lee IM, Gaziano JM, Glynn RJ. Metabolic abnormalities and risk for colorectal cancer in the physicians' health study. Cancer Epidemiol Biomarkers Prev. 2006; 15: 2391-7.

20. Russo A, Autelitano M, Bisanti L. Metabolic syndrome and cancer risk. Eur J Cancer. 2008; 44: 293-7.

21. Inoue $M$, Noda $M$, Kurahashi N, Iwasaki M, Sasazuki S, Iso H, et al. Impact of metabolic factors on subsequent cancer risk: results from a large-scale population-based cohort study in Japan. Eur J Cancer Prev. 2009; 18: 240-7.

22. Osaki Y, Taniguchi S, Tahara A, Okamoto M, Kishimoto T. Metabolic syndrome and incidence of liver and breast cancers in Japan. Cancer Epidemiol. 2012; 36: 141-7.

23. Ko S, Yoon SJ, Kim D, Kim AR, Kim EJ, Seo HY. Metabolic Risk Profile and Cancer in Korean Men and Women. Journal of preventive medicine and public health $=$ Yebang Uihakhoe chi. 2016; 49: 143-52.

24. Watanabe J, Kakehi E, Kotani K, Kayaba K, Nakamura Y, Ishikawa S. Metabolic syndrome is a risk factor for cancer mortality in the general Japanese population: the Jichi Medical School Cohort Study. Diabetol Metab Syndr. 2019; 11: 3.

25. Stocks T, Lukanova A, Bjorge T, Ulmer H, Manjer J, Almquist M, et al. Metabolic factors and the risk of colorectal cancer in 580,000 men and women in the metabolic syndrome and cancer project (Me-Can). Cancer. 2011; 117: 2398-407.

26. Matthews CE, Sui X, LaMonte MJ, Adams SA, Hebert JR, Blair SN. Metabolic syndrome and risk of death from cancers of the digestive system. Metabolism. 2010; 59: 1231-9.

27. Yang Y, Mauldin PD, Ebeling M, Hulsey TC, Liu B, Thomas MB, et al. Effect of metabolic syndrome and its components on recurrence and survival in colon cancer patients. Cancer. 2013; 119: 1512-20.

28. Esposito K, Chiodini P, Capuano A, Bellastella G, Maiorino MI, Rafaniello C, et al. Colorectal cancer association with metabolic syndrome and its components: a systematic review with meta-analysis. Endocrine. 2013; 44: 634-47.

29. Giri S, Grimshaw A, Bal S, Godby K, Kharel P, Djulbegovic B, et al. Evaluation of Daratumumab for the Treatment of Multiple Myeloma in Patients With High-risk Cytogenetic Factors: A Systematic Review and Meta-analysis. JAMA Oncol. 2020.

30. Hardy RJ, Thompson SG. Detecting and describing heterogeneity in meta-analysis. Statistics in medicine. 1998; 17: 841-56.

31. DerSimonian R, Laird N. Meta-analysis in clinical trials. Controlled clinical trials. 1986; 7: 177-88.

32. Begg CB, Mazumdar M. Operating characteristics of a rank correlation test for publication bias. Biometrics. 1994; 50: 1088-101.

33. Seagroatt V, Stratton I. Bias in meta-analysis detected by a simple, graphical test. Test had 10\% false positive rate. Bmj. 1998; 316: 470; author reply -1 .

34. Bowers K, Albanes D, Limburg P, Pietinen P, Taylor PR, Virtamo J, et al. A prospective study of anthropometric and clinical measurements associated with insulin resistance syndrome and colorectal cancer in male smokers. Am J Epidemiol. 2006; 164: 652-64
35. Kabat GC, Kim MY, Peters U, Stefanick M, Hou LF, Wactawski-Wende J, et al. A longitudinal study of the metabolic syndrome and risk of colorectal cancer in postmenopausal women. European Journal Of Cancer Prevention. 2012; 21: 326-32.

36. Liang X, Margolis KL, Hendryx M, Rohan TE, Groessl EJ, Thomson CA, et al. Metabolic phenotype and risk of colorectal cancer in normal-weight postmenopausal women. Cancer Epidemiology Biomarkers and Prevention. 2017; 26: 155-61.

37. Choi YJ, Lee DH, Han KD, Shin CM, Kim N. Abdominal obesity, glucose intolerance and decreased high-density lipoprotein cholesterol as components of the metabolic syndrome are associated with the development of colorectal cancer. European journal of epidemiology. 2018; 33: 1077-85.

38. Li X, Chen H, Wang G, Feng X, Lyu Z, Wei L, et al. Metabolic Syndrome Components and the Risk of Colorectal Cancer: A Population-Based Prospective Study in Chinese Men. Frontiers in oncology. 2019; 9: 1047.

39. Stocks T, Lukanova A, Johansson M, Rinaldi S, Palmqvist R, Hallmans G, et al. Components of the metabolic syndrome and colorectal cancer risk; a prospective study. International journal of obesity. 2008; 32: 304-14.

40. Pelucchi C, Negri E, Talamini R, Levi F, Giacosa A, Crispo A, et al. Metabolic syndrome is associated with colorectal cancer in men. European journal of cancer. 2010; 46: 1866-72.

41. Aleksandrova $\mathrm{K}$, Boeing $\mathrm{H}$, Jenab M, Bueno-de-Mesquita HB, Jansen E, van Duijnhoven FJB, et al. Metabolic Syndrome and Risks of Colon and Rectal Cancer: The European Prospective Investigation into Cancer and Nutrition Study. Cancer Prevention Research. 2011; 4: 1873-83.

42. Li L, Huang HL, Zhang XY, Zhang XY, Lv ZH. [Association of metabolic syndrome and colorectal cancer]. Zhonghua wei chang wai ke za zhi = Chinese journal of gastrointestinal surgery. 2011; 14: 948-51.

43. Ulaganathan V, Kandiah M, Zalilah MS, Faizal JA, Fijeraid H, Normayah K, et al. Colorectal cancer and its association with the metabolic syndrome: a Malaysian multi-centric case-control study. Asian Pacific journal of cancer prevention : APJCP. 2012; 13: 3873-7.

44. Kontou N, Psaltopoulou T, Soupos N, Polychronopoulos E, Xinopoulos D, Linos A, et al. Metabolic syndrome and colorectal cancer: the protective role of Mediterranean diet--a case-control study. Angiology. 2012; 63: 390-6.

45. Milano A, Bianco MA, Buri L, Cipolletta L, Grossi E, Rotondano G, et al. Metabolic syndrome is a risk factor for colorectal adenoma and cancer: a study in a White population using the harmonized criteria. Therapeutic Advances in Gastroenterology. 2019; 12.

46. Shen ZL, Ye YJ, Bin LA, Yin MJ, Yang XD, Jiang KW, et al. Metabolic syndrome is an important factor for the evolution of prognosis of colorectal cancer: survival, recurrence, and liver metastasis. American Journal Of Surgery. 2010; 200: 59-63.

47. Cespedes Feliciano EM, Kroenke $\mathrm{CH}$, Meyerhardt JA, Prado CM, Bradshaw PT, Dannenberg AJ, et al. Metabolic Dysfunction, Obesity, and Survival Among Patients With Early-Stage Colorectal Cancer. J Clin Oncol. 2016; 34: 3664-71.

48. Peng $\mathrm{F}, \mathrm{Hu} \mathrm{D}$, Lin $\mathrm{X}$, Chen $\mathrm{G}$, Liang $\mathrm{B}$, Zhang $\mathrm{H}$, et al. Preoperative metabolic syndrome and prognosis after radical resection for colorectal cancer: The Fujian prospective investigation of cancer (FIESTA) study. International journal of cancer. 2016; 139: 2705-13.

49. Croft B, Reed M, Patrick C, Kovacevich N, Voutsadakis IA. Diabetes, Obesity, and the Metabolic Syndrome as Prognostic Factors in Stages I to III Colorectal Cancer Patients. J Gastrointest Cancer. 2019; 50: 221-9.

50. Chen DZ, Ji FY, Xu QM, Wu XX, Cai C, Zhang LJ, et al. Interaction of smoking and metabolic syndrome in increasing the recurrence risk of colorectal cancer in a Chinese male cohort: a retrospective study. Scientific reports. 2018; 8: 972.

51. Mafiana RN, Al-Kindi MS, Mafiana N, Al Lawati AS, Al Moundhri M. Impact of Metabolic Syndrome Diagnosis and Its Treatment on Survival of Colorectal Cancer Patients. J of Cancer Epidemiology. 2019; 2019(4):1-9.

52. You J, Liu WY, Zhu GQ, Wang OC, Ma RM, Huang GQ, et al. Metabolic syndrome contributes to an increased recurrence risk of non-metastatic colorectal cancer. Oncotarget. 2015; 6: 19880-90.

53. You J, Zhang H, Shen Y, Chen C, Liu W, Zheng M, et al. Impact of platelet to lymphocyte ratio and metabolic syndrome on the prognosis of colorectal cancer patients. Mediators Inflamm. 2017; 10: 2199-208.

54. Ahmadi A, Noroozi M, Pourhoseingholi MA, Hashemi-Nazari SS. Effect of metabolic syndrome and its components on survival in colorectal cancer: a prospective study. Journal of renal injury prevention. 2015; 4 : 15-9.

55. Hu D, Zhang M, Zhang H, Xia Y, Lin J, Zheng X, et al. Prediction of Metabolic Syndrome for the Survival of Patients With Digestive Tract Cancer: A Meta-Analysis. Front Oncol. 2019; 9: 281.

56. Ulaganathan V, Kandiah M, Mohd Shariff Z. A case-control study of the association between metabolic syndrome and colorectal cancer: a comparison of International Diabetes Federation, National Cholesterol Education Program Adults Treatment Panel III, and World Health Organization definitions. Journal of gastrointestinal oncology. 2018; 9: 650-63.

57. Aleksandrova K, Jenab M, Bueno-de-Mesquita HB, Fedirko V, Kaaks R, Lukanova A, et al. Biomarker patterns of inflammatory and metabolic pathways are associated with risk of colorectal cancer: results from the European Prospective Investigation into Cancer and Nutrition (EPIC). European journal of epidemiology. 2014; 29: 261-75.

58. Iwatsuki M, Mimori K, Yokobori T, Ishi H, Beppu T, Nakamori S, et al. Epithelial-mesenchymal transition in cancer development and its clinical significance. Cancer science. 2010; 101: 293-9. 
59. Zhou C, Qian W, Li J, Ma J, Chen X, Jiang Z, et al. High glucose microenvironment accelerates tumor growth via SREBP1-autophagy axis in 2019; 38: 302.

60. Stattin P, Bjor O, Ferrari P, Lukanova A, Lenner P, Lindahl B, et al. Prospective study of hyperglycemia and cancer risk. Diabetes care. 2007; 30: 561-7. 